\title{
Comparison between Hydroponically and Conventionally and Organically Grown Lettuces for Taste, Odor, Visual Quality and Texture: A Pilot Study
}

\author{
Matthew T. Murphy, Fannie Zhang, Yukiko K. Nakamura, Stanley T. Omaye
}

Department of Nutrition, University of Nevada, Reno, USA.

Email: omaye@unr.edu

Received October 21 $1^{\text {st }}, 2010$; revised November $18^{\text {th }}, 2010$; accepted December $26^{\text {th }}, 2010$.

\begin{abstract}
Hydroponic production of vegetables is becoming more common. In this study, hydroponic lettuce grown by a local distributor and conventionally and organically field-grown lettuces purchased at local retail stores were compared by descriptive analysis for taste, odor, visual quality and texture. Five lettuce varieties were compared: Romaine, Green Leaf, Red Leaf, Butter and Common lettuce. A twenty-three member sensory panel randomly rated the lettuces using a 5 point scale or a 3 point scale for taste, odor, visual quality and texture. Analysis of variance (ANOVA) was performed for each lettuce variety with comparison between those hydroponically, organically and conventionally grown. Overall, panel members equally liked the different lettuce samples. The results showed that for each of the five varieties of lettuces, all lettuces were perceived to be equal in their sensory evaluation for those grown locally and hydroponically or purchased from local grocery as organically or conventionally grown.
\end{abstract}

Keywords: Hydroponic, Lettuce, Leafy Vegetables, Vegetables, Sensory

\section{Introduction}

People are increasingly interested in eating diets high in fruits and vegetables because of the purported health benefits. Extensive research has provided evidence showing strong inverse correlations between fruits and vegetable consumption and the risk of many types of chronic disease, e.g., cancer, cardiovascular diseases, and neurological disorders [1-6]. Several components or bioactive compounds found in fruits and vegetables are likely the source of such health benefits. Bioactive nutrients, such as beta-carotene and polyphenols, can be defined as constituents in foods, other than those needed to meet basic human nutritional needs that are responsible for beneficial effects in health status (Federal Register, Vol. 69, No. 179, 2004, pp. 55821-55822). This definition includes certain essential nutrients, whereby intake beyond what will reduce the risk of deficiency has been shown to have additional health benefits.

It is widely accepted that the quality (sensory perception and bioactive nutrient content) of fruits and vegeta- bles can be influenced by several key factors, such as genotype selection and environmental conditions (light, temperature, geography, cultivars, drought resistance, humidity, atmospheric $\mathrm{CO}_{2}$, and pollutants in the air) [7]. Over time and through genotype selection, hybrid cultivars have been developed with a high yield potential, uniform and pleasing appearance, and increased disease tolerance. Because of increased interest, the content of health promoting compounds is becoming a vital consideration for fruit and vegetable producers and growers. By means of plant-breeding programs, we can enhance levels of carotenoids [8] and other antioxidants [9]. For example, one can enhance the purple-fruitiness of tomatoes, increasing the level of the bioactive compound, anthocynanins [10].

There can be a high degree of control over the environment using greenhouse technology which can affect the perceived quality of fruits and vegetables. The objective of this study was to estimate the sensory evaluation of hydroponic lettuce compared to organically and conventionally grown products. 


\section{Materials and Methods}

\subsection{Lettuce Production}

Five lettuce varieties were selected on the bases of avail ability for testing and included; Variety 1 which was Romaine (cos lettuce), Variety 2 which was Green Leaf (loose leaf lettuce), Variety 3 which was Red Leaf (loose leaf lettuce), Variety 4 which was Butter (butter head lettuce), and Variety 5 which was Common Lettuce (green lettuce, Lactuca sativa).

Lettuce was grown hydroponically under greenhouse conditions at minimum and maximum temperatures of 13 and $24^{\circ} \mathrm{C}$ (Nevada Naturals, University of Nevada Field Station). Plants were grown in fiber pots containing perlite using a Hydroponics Nutrient Film Technique Kit. Water and nutrients were recirculated through the hydroponic unit from reservoir using a pump with a recycle rate of 1 liter/minute. Once seeds had germinated, tap water was replaced with a commercially available nutrient solution based on the report by Hochmuth and Hochmuth [11], and with maintenance of the $\mathrm{pH}$ between 5 and 6. At harvest, aerial portions of lettuce were harvested by removing vegetation above the perlite growing medium. Conventionally and organically grown lettuces were purchased at local retail stores (Scolari's Grocery, Reno, NV). Plant material was washed first in tap water and subsequently twice with distilled water to remove any particles.

\subsection{Sample Preparation for Sensory Evaluation}

Five heads of lettuces were provided from each source. Heads of lettuces were washed and rinsed once with running tap water twice with distilled water and excess water was removed by placing the lettuces in plastic colander strainer (1 hour). Lettuces were kept in a refrigerator/cold box $\left(4^{\circ} \mathrm{C}\right)$ until the experiment was performed.

Prior to the experiment, bit-sized samples (20 g) were placed in identical Dixie cups labeled with code numbers representing the sources of lettuces. Volunteers were provided with samples in Dixie cups and asked to taste each sample twice, rinsing their mouth with bottle water between each sample. They were asked to rate lettuce samples from 1 to 5 for taste from no taste to very intense or distinct taste. They were asked to rate whether the taste was sweet, bitter, salty, or sour and judge odor, visual qualities and texture. Volunteers also indicated on the score sheets their gender and age.

Data was tabulated and the averages were calculated and analyzed statistically to determine whether there was a difference in people's taste of hydroponic grown lettuce compared to store-purchased lettuces. The influence of gender and age was also analyzed.

\subsection{Panel Sensory Analysis}

Sensory evaluation of lettuce samples determined texture, flavor, color and overall acceptability using a quantitative descriptive analysis. The panel included 19 female and 4 male members, ages 20 to 52 with a mean age of 26 years who were participants in a food science class at the University. There was no information collected to link individual identities to sensory perception data. The students had classroom training and previous experience as judges in sensory evaluation for other foods, including participant bias and how to avoid bias, such as oral and visual communications between participants. Two sessions were conducted, a preliminary session to acquaint the participants with the laboratory and surroundings followed by the judging session. The lettuce samples were presented at room temperature to the participants. Each judge evaluated the intensity of the attribute, in a score sheet based on 5 point scales, including a simple "smiley face" scale which depicted cartoon faces from

Table 1. Mean visual quality and texture evaluation of hydroponically, organically and conventionally grown lettuces.

\begin{tabular}{ccccc}
\hline Lettuce variety & Visual quality & Pr $>$ F & Texture & Pr $>$ F \\
\hline Variety 1 & & & & \\
Hydroponically & $2.3 \pm 1.2$ & & $2.3 \pm 1.2$ & \\
Organically & $3.1 \pm 1.4$ & 0.0678 & $2.7 \pm 1.2$ & 0.3282 \\
Conventionally & $3.0 \pm 1.4$ & & $2.8 \pm 1.0$ & \\
$\quad$ Variety 2 & & & & \\
Hydroponically & $3.4 \pm 1.3$ & & $3.2 \pm 1.2$ & \\
Organically & $3.2 \pm 1.2$ & 0.7559 & $3.0 \pm 1.1$ & 0.7199 \\
Conventionally & $3.5 \pm 1.2$ & & $3.1 \pm 1.0$ & \\
$\quad$ Variety 3 & & & & \\
Hydroponically & $4.1 \pm 1.1$ & & $3.2 \pm 1.2$ & \\
Organically & $3.8 \pm 1.2$ & 0.526 & $3.3 \pm 1.3$ & 0.5259 \\
Conventionally & $4.1 \pm 1.1$ & & $3.6 \pm 1.0$ & \\
$\quad$ Variety 4 & & & & \\
Hydroponically & $4.1 \pm 0.9$ & & $3.0 \pm 1.4$ & \\
Organically & $4.0 \pm 0.9$ & 0.8701 & $3.1 \pm 1.2$ & 0.9716 \\
Conventionally & $4.0 \pm 1.0$ & & $3.1 \pm 1.1$ & \\
$\quad$ Variety 5 & & & & \\
Hydroponically & $3.9 \pm 1.1$ & & $3.0 \pm 1.3$ & \\
Organically & $4.0 \pm 1.0$ & 0.9467 & $2.9 \pm 1.3$ & 0.9349 \\
Conventionally & $4.0 \pm 1.0$ & & $3.1 \pm 1.2$ & \\
\hline & & &
\end{tabular}

Visual quality and texture means (based on 5 point observational scale) between hydroponically, organically and conventionally grown lettuces for: Variety 1 (Romaine), 2 (Green Leaf), 3 (Red Leaf), 4 (Butter) and 5 (Common lettuce), respectively.

Data are means and standard deviations of 23 judges' evaluations. Pr > F (o-value associated with F Statistic of a given source). 
Table 2. Mean odor and odor character evaluation of hydroponically, organically and conventionally grown lettuces.

\begin{tabular}{lcccc}
\hline Lettuce & Odor & Pr $>$ F & Odor character & Pr $>$ F \\
\hline Variety 1 & & & & \\
Hydroponically & $2.4 \pm 1.4$ & & $2.1 \pm 0.9$ & \\
Organically & $1.9 \pm 0.9$ & 0.2709 & $2.8 \pm 0.5$ & 0.0207 \\
Conventionally & $2.0 \pm 1.1$ & & $2.6 \pm 0.8$ & \\
Variety 2 & & & & \\
Hydroponically & $2.0 \pm 1.2$ & & $2.5 \pm 0.9$ & \\
Organically & $2.3 \pm 1.4$ & 0.7492 & $2.8 \pm 0.9$ & 0.8677 \\
Conventionally & $2.3 \pm 1.1$ & & $2.3 \pm 0.9$ & \\
Variety 3 & & & & \\
Hydroponically & $2.1 \pm 1.2$ & & $2.5 \pm 0.8$ & \\
Organically & $2.3 \pm 1.4$ & 0.8257 & $2.7 \pm 0.7$ & 0.6694 \\
Conventionally & $2.2 \pm 1.2$ & & $2.5 \pm 0.8$ & \\
Variety 4 & & & & \\
Hydroponically & $1.9 \pm 1.1$ & & $2.7 \pm 0.6$ & \\
Organically & $2.1 \pm 1.2$ & 0.7952 & $2.4 \pm 0.8$ & 0.2705 \\
Conventionally & $2.1 \pm 1.2$ & & $2.7 \pm 0.6$ & \\
Variety 5 & & & & \\
Hydroponically & $2.3 \pm 1.4$ & & $2.3 \pm 0.8$ & \\
Organically & $2.0 \pm 0.2$ & 0.467 & $2.2 \pm 0.9$ & 0.439 \\
Conventionally & $2.5 \pm 1.9$ & & $2.5 \pm 0.8$ & \\
\hline OD & & & \\
\hline
\end{tabular}

Odor means (based on 5 point observational scale) and odor character means (based 1 = bitter, 2 = sweet, and 3 = other) between hydroponically, organically and conventionally grown lettuces for: Variety 1 (Romaine), 2 (Green Leaf), 3 (Red Leaf), 4 (Butter) and 5 (Common lettuce), respectively.

Data are means and standard deviations of 23 judges' evaluations. Pr > F (o-value associated with F Statistic of a given source).

smiles to frowns [12], for which they were instructed that the cartoons went from positive/favorable evaluations to negative/unfavorable evaluations, respectively (as an attempt to remove culture connotations of the cartoons). The following attributes were evaluated: odor, visual qualities, taste (sweetness, sour, salty or bitter) and texture.

\subsection{Statistical Analysis}

Data from volunteer testing were analyzed using SAS ${ }^{\circledR}$ (Version 9.1, Cry, N.C., USA). Analysis of variance (ANOVA) was performed for each lettuce variety with comparison between those grown hydroponically, organically and conventionally grown. $\mathrm{df}=2$. Significant differences were determined at the $95 \%$ confidence level $(\mathrm{p}<0.05)$.

\section{Results}

Before sensory analysis of hydroponically, organically
Table 3. Mean taste and taste character evaluation of hydroponically, organically and conventionally grown lettuces.

\begin{tabular}{ccccc}
\hline Lettuce & Taste & Pr $>$ F & $\begin{array}{c}\text { Taste } \\
\text { character }\end{array}$ & Pr $>$ F \\
\hline Variety 1 & & & & \\
Hydroponically & $3.2 \pm 1.3$ & & $1.8 \pm 0.9$ & \\
Organically & $2.6 \pm 1.2$ & 0.2614 & $2.1 \pm 0.9$ & 0.5242 \\
Conventionally & $3.0 \pm 1.2$ & & $1.9 \pm 1.0$ & \\
Variety 2 & & & & \\
Hydroponically & $3.2 \pm 1.2$ & & $1.9 \pm 0.9$ & \\
Organically & $3.0 \pm 1.4$ & 0.8374 & $1.9 \pm 1.0$ & 0.9365 \\
Conventionally & $3.0 \pm 1.3$ & & $2.0 \pm 1.0$ & \\
Variety 3 & & & & \\
Hydroponically & $3.4 \pm 1.3$ & & $2.1 \pm 0.8$ & \\
Organically & $3.3 \pm 1.3$ & 0.9652 & $1.8 \pm 0.9$ & 0.4763 \\
Conventionally & $3.4 \pm 1.3$ & & $1.9 \pm 0.9$ & \\
Variety 4 & & & & \\
Hydroponically & $3.5 \pm 1.3$ & & $1.9 \pm 0.7$ & \\
Organically & $3.2 \pm 1.1$ & 0.7207 & $1.9 \pm 0.9$ & 0.9249 \\
Conventionally & $3.4 \pm 1.1$ & & $1.8 \pm 0.9$ & \\
$\quad$ Variety 5 & & & & \\
Hydroponically & $3.5 \pm 1.5$ & & $1.9 \pm 0.9$ & \\
Organically & $3.3 \pm 1.2$ & 0.5976 & $1.7 \pm 0.8$ & 0.6863 \\
Conventionally & $3.1 \pm 1.2$ & & $2.0 \pm 0.9$ & \\
\hline
\end{tabular}

Means (based on 5 point observational scale) and taste character means (based 1 = bitter, 2 = sweet, and 3 = other) with the same letter in a column are not significantly different between hydroponically, organically and conventionally grown lettuces for: Variety 1 (Romaine), 2 (Green Leaf), 3 (Red Leaf), 4 (Butter) and 5 (Common lettuce), respectively.

Data are means and standard deviations of 23 judges' evaluations. Pr > F (o-value associated with F Statistic of a given source).

and conventionally grown lettuce does not show any significant difference with respect to visual quality, texture, odor, taste or taste character (Table 1-3).

There was a significant difference among the three groups for Variety $1(\mathrm{p}=0.0207)$ shown in Table 2 . A significant difference was found either between hydroponically and conventionally grown lettuces $(p=0.03)$ or between organically and conventionally grown lettuces $(p=0.009)$, but not between hydroponically and organically grown lettuces $(p=0.6956)$. The perception of the panel was that hydroponically and organically grown lettuce had an odor.

\section{Discussion}

Although this study had methodological limitations, participants gained experience on the basic elements of being a sensory panel member. They were instructed on the potential bias that might occur and what was necessary to avoid such bias; thus, providing a more informed evalua- 
tion of the lettuce samples.

Nutritional quality of fruits and vegetables can be manipulated by changes in light and temperature. Higher altitudes reduce temperature and increase visible and UV exposure, which in turn increases plant carotenoid content. This could have been a factor in the lettuce products produced locally in Reno, Nevada at an altitude of 4,500 feet. Vitamin C, carotenoids, anthocyanin, and phenols are strongly affected by light intensity [13-15]. For changes in plant vitamin $\mathrm{C}$, this bioactive compound is synthesized from sugars. The amount and intensity of light during the growing season affect sugar synthesized via photosynthesis [14]. For other bioactive compounds, sufficient energy of light is important to promote the photosynthetic production of carbohydrates, which in turn are the substrates for flavonoid biosynthesis via the shikimic acid and phenylpropanoid pathways. Temperature has a direct influence on plant metabolism. Low ambient temperatures can result in higher levels synthesis of photochemical synthesis. Vitamin C levels generally decline with increasing temperatures and some levels of bioactive compounds are increased when temperature are optimal, $12^{\circ} \mathrm{C}$ to $21^{\circ} \mathrm{C}$.

In conclusion, there are many parameters that could have impacted on perceived sensory difference for the lettuces tested in our study. Five varieties of lettuces, those locally and hydroponically grown or purchased from local grocery outlets, were evaluated by a panel for taste, odor, visual qualities and texture. No differences were found in the sensory evaluations between the lettuces types, irrespectively from how they were grown.

\section{Acknowledgements}

We appreciate the Nevada Agricultural Experimental Station and hydroponic lettuce donations from Tom and John Blount of Nevada Naturals.

\section{REFERENCES}

[1] S. T. Mayne, "Beta-carotene, Carotenoids and Disease Prevention in Humans,” The FASEB Journal, Vol. 10, No. 7, 1996, pp. 690-701.

[2] S. T. Omaye and P. Zhang, "Phytochemical Interactions: Beta-carotene, Tocopherol and Ascorbic Acid,” In: Phytochemicals: A New Paradigm. W. R. Bidlack, S. T. Omaye, M. S. Meskin and D. Jahner, Eds., Technomic Publishing Co., 1996, pp. 53-76.

[3] M. A. Dubick and S. T. Omaye, "Evidence for Grape, Wine and Tea Polyphenols as Modulators of Arteriosclerosis and Ischemic Heart Disease in Humans," Nutraceuticals, Functional \& Medical Foods, Vol. 3, No. 3, 2001, pp. 67-93.

[4] E. Giovannucci, "A Review of Epidemiologic Studies of Tomatoes, Lycopene, and Prostate Cancer,” Experimental Biology and Medicine, Vol. 227, No. 10, 2002, pp. 852-859.

[5] E. Giovannucci, E. Rimm, Y. Liu, M. Stampfer and W. A. Willett, "A Prospective Study of Tomato Products, Lycopene, and Prostate Cancer Risk," Journal of the National Cancer Institute, Vol. 94, No. 5, 2002, pp. 391-398.

[6] M. Dorais, D. L. Ehret and A. P. Papadopoulos, "Tomato (Solanum Lycopersicum) Health Components: From Seed to the Consumer," Phytochemistry Reviews, Vol. 7, No. 2, 2008, pp. 231-250. doi:10.1007/s11101-007-9085-X

[7] A. K. Thybo, M. Edelenbos, L. P. Christensen, J. Sorensen and K. Thorup-Kristensen, "Effect of Organic Growing Systems on Sensory Quality and Chemical Composition of Tomatoes," Food Science and Technology, Vol. 39, No. 8, 2005, pp. 835-843.

[8] G. Ronen, L. Carmel-Goren, D. Zamir and J. Hirschberg, "An Alternative Pathway to Beta-Carotene Formation in Plant Chromoplast Discovered by Map-Based Cloning of Beta (B) and Old-Gold (Or) Color Mutations in Tomato," Proceedings of the National Academy of Sciences, Vol. 97, No. 20, 2000, pp. 11102-11107. doi:10.1073/pnas.190177497

[9] E. G. Schijlen, C. H. Ric de Vos, A. J. Van Tunen and A. G. Bovy, "Modification of Flavonoid Biosynthesis in Crop Plants,” Phytochemistry, Vol. 65, No. 19, 2004, pp. 2631-2648. doi:10.1016/j.phytochem.2004.07.028

[10] C. M. Jones, P. Mes and J. R. Myers, "Characterization and Inheritance of the Antocyanin Fruit (Aft) Tomato," Journal of Heredity, Vol. 94, No. 6, 2003, pp 449-456. doi:10.1093/jhered/esg093

[11] G. Hochmuth and R. C. Hochmuth, "Nutrient Solution Formulation for Hydroponic (Perlite, Rockwood, NFT) Tomatoes in Florida," University o Florida IFAS Extension, Report 1996, \# HS796.

[12] H. R. Moskowitz, J. H. Beckley and A. V. A. Resurreccion, "Sensory and Consumer Research in Food Product Design and Development,” Blackwell Publishing, 2006. doi:10.1002/9780470277706

[13] J. P. McCollum, "Effects of Light on the Formation of Carotenoids in Tomato Fruit," Food Research, Vol. 19, 1954, pp. 182-189.

[14] K. S. Lee and A. A. Kader, "Preharvest and Postharvest Factors Influencing Vitamin C Content of Horticultural Crops," Postharvest Biology and Technology, Vol. 20, No. 3, 2000, pp. 207-220. doi:10.1016/S0925-5214(00)00133-2

[15] J. T. Amiot, F. Tourniaire and A. Margotat, "Flavonoids in Food and Wine,” Acta Horticulture, Vol. 744, 2007, pp. 107-116. 